Review began 02/26/2022 Review ended 02/26/2022 Published 02/28/2022

๑) Copyright 2022

Gurjar et al. This is an open access article distributed under the terms of the Creative Commons Attribution License CC-BY 4.0. which permits unrestricted use, distribution, and reproduction in any medium, provided the original author and source are credited.

\section{A High-Risk Patient With COVID-19 Vaccine Hesitancy Successfully Treated With Monoclonal Antibodies Through Two Major Surges}

\author{
Hitesh Gurjar ${ }^{1}$, Haider Ghazanfar ${ }^{1}$, Asim Haider ${ }^{1}$, Nolberto Hernandez ${ }^{1}$, Abhilasha Jyala ${ }^{1}$, Sridhar
} Chilimuri $^{2}$

1. Internal Medicine, BronxCare Health System, Bronx, USA 2. Internal Medicine/Gastroenterology, BronxCare Health System, Bronx, USA

Corresponding author: Haider Ghazanfar, haidergh@gmail.com

\begin{abstract}
Vaccine hesitancy remains a significant challenge in managing the current pandemic despite highly effective vaccines in the United States. Monoclonal antibodies $(\mathrm{mAb})$ are an essential addition to coronavirus disease 2019 (COVID-19) treatment, along with oral antiviral agents (OAA), for non-hospitalized patients having risk factors for progression to severe COVID-19, especially in unvaccinated people. We present a case of a 74-year-old unvaccinated Hispanic woman with a history of diabetes mellitus, hypertension, coronary artery disease, obesity, and asthma who survived two episodes of severe acute respiratory syndrome coronavirus 2 (SARS-CoV-2) infections in January 2021 and December 2021 with exclusive use of mAb. Our case highlights the importance of using mAbs for treating high-risk patients with SARS-CoV-2 infection, especially in patients with vaccine hesitancy.
\end{abstract}

Categories: Internal Medicine, Infectious Disease

Keywords: covid-19, vaccine hesitancy, sotrovimab, bamlanivimab, monoclonal antibodies

\section{Introduction}

Despite the wide availability of highly effective coronavirus disease 2019 (COVID-19) vaccines, the United States continues to see major surges of severe acute respiratory syndrome coronavirus 2 (SARS-CoV-2) infections due to vaccine hesitancy. The CDC reports that unvaccinated patients account for a vast majority of SARS-CoV-2 deaths. Monoclonal antibodies (mAb) are an important addition to COVID-19 treatment along with oral antiviral agents (OAA) for non-hospitalized patients with risk factors for progression to severe COVID-19 [1].

These antibodies bind to the spike protein of SARS-CoV-2 and prevent its entry and further disease worsening [2]. They are currently approved for use in adult and pediatric patients, who are $\geqslant 12$ years old and weigh at least $40 \mathrm{~kg}$, and tested positive for SARS-CoV-2 with risk factors for progression of the disease to severe COVID-19. These are not authorized for hospitalized patients, patients requiring oxygen, or increasing oxygen requirements for those on long-term oxygen therapy. We present a case of a 74-year-old unvaccinated Hispanic woman who survived two episodes of COVID-19 infection with mAbs, despite multiple major risk factors, demonstrating the value of mAb. Resistance to mAb has emerged, with only sotrovimab and bebtelovimab remaining effective against the omicron variant of SARS-CoV-2.

\section{Case Presentation}

Our patient is a 74-year-old Hispanic woman who first presented in January 2021 with body aches, headache, loss of smell, change in taste, dry cough, and fever of five-day duration. Her past medical history was significant for diabetes mellitus, hypertension, coronary artery disease, obesity, and asthma. Past surgical history was significant for coronary artery bypass, hysterectomy, and cystocele repair. Her family history was remarkable for hypertension and diabetes. She had no known drug allergies. She denied smoking, alcohol abuse, or use of recreational drugs. She had not received any COVID-19 vaccine. Her nasopharyngeal polymerase chain reaction (PCR) swab was positive for SARS-CoV-2. On physical examination, her temperature was 98.4 Fahrenheit $(\mathrm{F})$, respiratory rate was 16 breaths per minute, a saturation of $97 \%$ on room air, pulse rate of 56 beats per minute, and blood pressure of $113 / 61 \mathrm{mmHg}$. Her body mass index (BMI) was $35.6 \mathrm{~kg} / \mathrm{m}^{2}$. She appeared lethargic on examination and had bilateral crackles on lung auscultation. Cardiac examination revealed normal S1 and S2 heart sounds. Abdominal and neurological examination was unremarkable. She was offered $\mathrm{mAb}$ infusion, given her several risk factors for disease progression. Her initial laboratory findings are presented in Table 1. Her SARS-CoV-2 nucleocapsid antibodies were non-reactive as detected by Elecsys Anti-SARS-CoV-2 test (Roche Diagnostics International Ltd., Rotkreuz, Switzerland). Since the dominant strain was Alpha variant she agreed and received bamlanivimab infusion. She did not experience any side effects from the mAb infusion. She reported that fever, body aches, headaches, loss of smell, and change in taste resolved after 24 hours of infusion. Her cough resolved on the third day after the mAb infusion. 


\section{Cureus}

One year later, she presented again with a dry cough, headache, fever, loss of smell, and change in taste for three days. She denied getting sick or hospitalized since the previous episode. She denied receiving any vaccination for COVID-19 in the interim. Her nasopharyngeal PCR swab was positive for SARS-CoV-2. At presentation, her temperature was $98.4 \mathrm{~F}$, respiratory rate was 15 breaths per minute, a saturation of $98 \%$ on room air, pulse rate of 72 beats per minute, and blood pressure of 144/91 mmHg. She had incessant coughing and appeared lethargic on general examination. She had bilateral vesicular breathing on lung auscultation. Cardiac examination revealed normal S1 and S2 heart sounds. Abdominal and neurological examination was unremarkable. Since she had multiple risk factors and worsening symptoms, she was offered mAb infusion. Her laboratory findings are summarized in Table 1.

\begin{tabular}{|c|c|c|c|}
\hline Laboratory Investigation & First Encounter & Second Encounter & Reference Range \\
\hline White Blood Cell Count (K/ul) & 4.0 & 6.1 & $4.8-10.8$ \\
\hline Absolute Lymphocyte Count (K/ul) & 2.5 & 2.4 & $1.0-4.8$ \\
\hline Hemoglobin (g/dl) & 11.8 & 11.5 & $12.0-16.0$ \\
\hline Hematocrit (\%) & 36.1 & 35.4 & $42.0-51.0$ \\
\hline Platelet count (K/ul) & 177 & 205 & $150-400$ \\
\hline Serum Sodium (mEq/l) & 133 & 136 & $135-145$ \\
\hline Serum Potassium (mEq/l) & 4.2 & 4.1 & $3.5-5.0$ \\
\hline Serum bicarbonate $(\mathrm{mEq} / \mathrm{l})$ & 31 & 27 & $24-30$ \\
\hline Serum Chloride (mEq/l) & 96 & 98 & $98-108$ \\
\hline Blood Urea Nitrogen (mEq/l) & 9.0 & 9.0 & $6.0-20.0$ \\
\hline Serum Creatinine (mEq/l) & 1.0 & 1.0 & $0.5-1.5$ \\
\hline Lactate Dehydrogenase (unit/L) & 211 & 371 & $110-210$ \\
\hline C-Reactive Protein (mg/L) & $<5.00$ & 13.31 & $\leq 5.00$ \\
\hline D-Dimer (ng/mL) & $<150$ & 157 & $0-230$ \\
\hline Ferritin (ng/mL) & 101. 0 & 77.9 & $13-150$ \\
\hline SARS-CoV-2 nucleocapsid antibodies & Non-reactive & Reactive & Non-Reactive \\
\hline SARS-CoV-2 spike antibodies & Not available & $22.50 \mathrm{U} / \mathrm{mL}$ & $>=0.00 \mathrm{U} / \mathrm{mL}$ \\
\hline
\end{tabular}

\section{TABLE 1: Summary of Laboratory Findings During Both Clinical Encounters}

Her SARS-CoV-2 nucleocapsid antibodies detected by Elecsys Anti-SARS-CoV-2 test (Roche Diagnostics International Ltd., Rotkreuz, Switzerland) were reactive and her SARS-CoV-2 spike antibody level (detected using Elecsys Anti SARS-CoV-2 S assay \{Roche Diagnostics International Ltd., Rotkreuz, Switzerland\}) were $22.50 \mathrm{U} / \mathrm{mL}$ (reference range > $=0.00 \mathrm{U} / \mathrm{mL}$ ). In June 2021, the Delta variant became dominant in New York, followed by the Omicron variant in December 2021, which has been reported to evade the immunity obtained from prior infection and vaccination. Sotrovimab was also reported as the only effective $\mathrm{mAb}$ for the Omicron variant. She received sotrovimab mAb infusion without any adverse effects. She reported symptom improvement after 24 hours of mAb infusion, with complete resolution after 48 hours of $\mathrm{mAb}$ infusion. She denied any adverse event or hospitalization on the 30th-day follow-up after infusion.

\section{Discussion}

Monoclonal antibodies bind to the spike protein of SARS-CoV-2 and prevent its entry and further disease worsening [2]. Bamlanivimab (November 9, 2020), bamlanivimab with etesevimab (February 9, 2021), casirivimab and imdevimab (November 21, 2020), sotrovimab (May 26, 2021) and bebtelovimab (February 11, 2022) were approved under emergency use authorization (EUA) by U.S. Food and Drug Administration (FDA) (parentheses include the date of EUA approval respectively). The timeline of EUA approval of mAbs and the emergence of the SARS-CoV-2 variant is depicted in Figure 1. 


\section{Cureus}

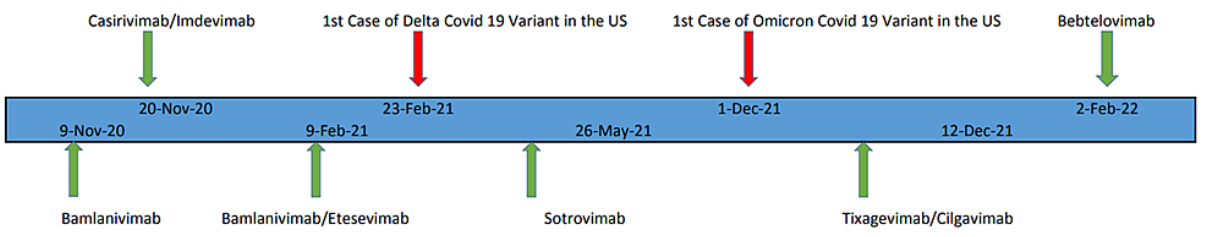

FIGURE 1: Timeline depicting Emergency Use Authorization of Monoclonal Antibodies and Emergence of SARS-CoV-2 Variant

SARS-CoV-2: Severe acute respiratory syndrome coronavirus 2

COVID 19: Coronavirus disease 2019

US: United States

Due to the emergence of resistance, the use of bamlanivimab as a single agent was revoked [3]. Subsequently, EUA has withdrawn for casirivimab and imdevimab due to lack of effectiveness against the Omicron variant. In December 2021, the Omicron variant became the predominant infection in New York. Sotrovimab is the only mAb effective against Omicron. First case of Omicron variant was detected on December 2, 2021, in New York City. Within five weeks, it became the predominant strain (isolated in $90 \%$ of samples) compared to the delta variant, which required 20 weeks to become the predominant strain [4].

As of January 22, 2022, 75.5\% of the U.S. population has been vaccinated [5]. However, this figure is lower in certain regions, such as the Bronx County, where our patient resides [6]. Vaccine hesitancy appears high in certain communities in the U.S. It has been reported in $30.2 \%$ and $41.6 \%$ of Hispanics and African American populations, respectively [7]. Likewise, the use of mAb is also reportedly very low in Hispanics and African Americans [8]. Our case illustrates the effectiveness of mAb in treating a patient with vaccine hesitancy in this specific population.

She was treated with bamlanivimab in January 2021. The protection provided by mAbs usually lasts for 90 days, after which vaccine is recommended [9]. In our case, she refused the COVID-19 vaccine but readily agreed to receive $\mathrm{mAb}$ (sotrovimab) in December 2021. This case highlights certain important considerations. The use of $\mathrm{mAb}$ is a safe and effective treatment for individuals with high-risk conditions for disease progression. Age over 65 (Odd ratio:6.01), Hispanic race (Odds Ratio: 1.35), diabetes mellitus (Odd Ratio: 3.68), coronary artery disease (Odds Ratio: 3.23), and obesity (Odds Ratio: 4.17) have been associated with increased risk of progression to severe illness [10-14].

Many mAbs have been used in a variety of different clinical conditions, and their use has been associated with short-term side effects such as hypersensitivity reactions and long-term side effects such as infections, cancers, autoimmune conditions, and organ-specific toxicities [15]. We have not seen any long-term adverse events in our own experience of nearly 100 patients [16].

Vaccine hesitancy remains a major concern in the United States. Unvaccinated patients remain the largest group of patients currently hospitalized and account for the largest group of mortalities [17]. While the reasons for vaccine hesitancy appear to be multifactorial, it should not preclude physicians from offering $\mathrm{mAbs}$ in patients with high-risk factors for the progression of the disease [18]. These mAbs are well tolerated and are highly effective, as demonstrated in our patient. While effective, mAbs should not be inferred as an alternative to the vaccine, as indeed, vaccinations remain the primary goal. Introduction of new long-acting $\mathrm{mAb}$ (tixagevimab + cilgavimab) may be used in patients as pre-exposure prophylaxis in patients with specific contraindication for vaccine or those who have anticipated inadequate response to vaccine due to immunosuppression [19]. It is possible that when ample supplies become readily available, these can be used in a wider population.

\section{Conclusions}

Vaccine hesitancy remains a major hurdle in the management of the pandemic. However, the availability of $\mathrm{mAb}$ should provide effective treatment in patients with risk factors for progression, especially in the unvaccinated. While the greater goal is to increase vaccination rates, unvaccinated patients with risk factors should be offered mAb to prevent the progression of the disease. Our case highlights the importance of offering $\mathrm{mAb}$ to patients earlier in the disease course as it is associated with better clinical outcomes. Despite being at high risk for progression to severe COVID-19, our patient did well on mAb infusions and adverse outcomes were avoided.

\section{Additional Information}




\section{Disclosures}

Human subjects: Consent was obtained or waived by all participants in this study. Conflicts of interest: In compliance with the ICMJE uniform disclosure form, all authors declare the following: Payment/services info: All authors have declared that no financial support was received from any organization for the submitted work. Financial relationships: All authors have declared that they have no financial relationships at present or within the previous three years with any organizations that might have an interest in the submitted work. Other relationships: All authors have declared that there are no other relationships or activities that could appear to have influenced the submitted work.

\section{References}

1. NIH COVID-19 Treatment Guidelines: Therapeutic Management of Non Hospitalized Adults With COVID 19 2022. (2022). Accessed: February 17, 2022: https://www.covid19treatmentguidelines.nih.gov/management/clinical-management/nonhospitalizedadults--therapeutic-man....

2. Copin R, Baum A, Wloga E, et al.: The monoclonal antibody combination REGEN-COV protects against SARS-CoV-2 mutational escape in preclinical and human studies. Cell. 2021, 184:3949-3961.e11. 10.1016/j.cell.2021.06.002

3. Coronavirus (COVID-19) Update: FDA Revokes Emergency Use Authorization for Monoclonal Antibody Bamlanivimab. (2021). Accessed: February 21, 2022: https://www.fda.gov/news-events/pressannouncements/coronavirus-covid-19-update-fda-revokes-emergency-use-authorizati....

4. New York City Department of Health and Mental Hygiene. Omicron Variant: NYC Report for January 13, 2022. (2022). Accessed: February 21, 2022: https://www1.nyc.gov/assets/doh/downloads/pdf/covid/omicronvariant-report-jan-13-22.pdf.

5. Centers for Disease Control and Prevention. COVID Data Tracker: COVID-19 Vaccinations in the United States 2022. (2022). Accessed: February 21, 2022: https://covid.cdc.gov/covid-datatracker/\#vaccinations_vacc-total-admin-rate-total. $\backslash$.

6. Centers for Disease Control and Prevention. COVID Data Tracker: Vaccinations in Bronx County, New York 2022. (2022). Accessed: February 21, 2022: https://covid.cdc.gov/covid-data-tracker/\#county-view? list_select_state=New+York\&data-type=Vaccinations \&metric=Admini....

7. Khubchandani J, Macias Y: COVID-19 vaccination hesitancy in Hispanics and African-Americans: A review and recommendations for practice. Brain Behav Immun Health. 2021, 15:100277. 10.1016/j.bbih.2021.100277

8. Centers for Disease Control and Prevention. Morbidity and Mortality Weekly Report (MMWR): Racial and Ethnic Disparities in Receipt of Medications for Treatment of COVID-19 - United States, March 2020August 2021. (2022). Accessed: February 21, 2022: https://www.cdc.gov/mmwr/volumes/71/wr/mm7103e1.htm.

9. Centers for Disease Control and Prevention. COVID-19 Vaccine FAQs for Healthcare Professionals . (2022). Accessed: February 21, 2022: https://www.cdc.gov/vaccines/covid-19/hcp/faq.html.

10. Liang C, Zhang W, Li S, Qin G: Coronary heart disease and COVID-19: a meta-analysis . Med Clin (Barc). 2021, 156:547-54. 10.1016/j.medcli.2020.12.017

11. Chang MC, Park YK, Kim BO, Park D: Risk factors for disease progression in COVID-19 patients . BMC Infect Dis. 2020, 20:445. 10.1186/s12879-020-05144-X

12. Zheng Z, Peng F, Xu B, et al.: Risk factors of critical \& mortal COVID-19 cases: a systematic literature review and meta-analysis. J Infect. 2020, 81:e16-25. 10.1016/j.jinf.2020.04.021

13. Magesh S, John D, Li WT, et al.: Disparities in COVID-19 outcomes by race, ethnicity, and socioeconomic status: a systematic-review and meta-analysis. JAMA Netw Open. 2021, 4:e2134147. 10.1001/jamanetworkopen.2021.34147

14. Chu Y, Yang J, Shi J, Zhang P, Wang X: Obesity is associated with increased severity of disease in COVID-19 pneumonia: a systematic review and meta-analysis. Eur J Med Res. 2020, 25:64. 10.1186/s40001-020-004649

15. Hansel TT, Kropshofer H, Singer T, Mitchell JA, George AJ: The safety and side effects of monoclonal antibodies. Nat Rev Drug Discov. 2010, 9:325-38. 10.1038/nrd3003

16. Chilimuri S, Mantri N, Gurjar H, et al.: Implementation and outcomes of monoclonal antibody infusion for COVID-19 in an inner-city safety net hospital: a South-Bronx experience. J Natl Med Assoc. 2022, 113:7015. 10.1016/j.jnma.2021.08.036

17. Centers for Disease Control and Prevention. Morbidity and Mortality Weekly Report. COVID-19 Incidence and Death Rates Among Unvaccinated and Fully Vaccinated Adults with and Without Booster Doses During Periods of Delta and Omicron Variant Emergence - 25 U.S. Jurisdictions, April 4-December 25, 2021. (2022). Accessed: February 22, 2022: https://www.cdc.gov/mmwr/volumes/71/wr/mm7104e2.htm.

18. Sowa P, Kiszkiel Ł, Laskowski PP, et al.: COVID-19 vaccine hesitancy in Poland-multifactorial impact trajectories. Vaccines (Basel). 2021, 9:10.3390/vaccines9080876

19. Coronavirus (COVID-19) Update: FDA Authorizes New Long-Acting Monoclonal Antibodies for Preexposure Prevention of COVID-19 in Certain Individuals. (2021). Accessed: February 23, 2022: https://www.fda.gov/news-events/press-announcements/coronavirus-covid-19-update-fda-authorizes-newlong-acting-monocl.... 